\title{
Dispersion Features of Conducting Sheath Helix Embedded Elliptical and Circular Fibers with Chiral Nihility Core
}

\author{
N. Iqbal, M. A. Baqir, and P. K. Choudhury \\ Institute of Microengineering and Nanoelectronics, Universiti Kebangsaan Malaysia (UKM), 43600 Bangi, Malaysia \\ Correspondence should be addressed to P. K. Choudhury; pankaj@ukm.edu.my
}

Received 18 September 2014; Revised 30 December 2014; Accepted 1 January 2015

Academic Editor: Yongqi Fu

Copyright (C) 2015 N. Iqbal et al. This is an open access article distributed under the Creative Commons Attribution License, which permits unrestricted use, distribution, and reproduction in any medium, provided the original work is properly cited.

\begin{abstract}
Discussions have been made of the electromagnetic wave propagation through optical fibers of elliptical and circular cross-sections with the loadings of conducting sheath helix at the core-clad interface with particular orientations. In both geometrical situations, the core section of fiber is assumed to be composed of chiral nihility medium, and the clad remains linear, homogeneous, isotropic, and nonmagnetic dielectric. Dispersion relations are deduced for both fiber structures, followed by the analysis of dispersion features considering a few low-order EH modes. Furthermore, the effects of conducting helix pitch angle on the features of wave propagation are also discussed.
\end{abstract}

\section{Introduction}

Chiral materials possess a unique feature of exhibiting optical activity. Linearly polarized light upon passing through such materials splits into two circularly polarized light waves, and the medium behaves as isotropic for these [1]. Such complex mediums with different structural designs have been studied extensively.

Chiral nihility material is another newly recognized class which, by definition, has zero permittivity and permeability but nonzero chirality [2]. The constitutive relations for isotropic chiral mediums are written as [3]

$$
\begin{aligned}
& \mathbf{D}=\varepsilon \mathbf{E}-j \kappa \sqrt{\epsilon_{0} \mu_{0}} \mathbf{H} \\
& \mathbf{B}=\mu \mathbf{H}+j \kappa \sqrt{\epsilon_{0} \mu_{0}} \mathbf{E},
\end{aligned}
$$

where $\varepsilon, \mu$, and $\kappa$ are, respectively, permittivity, permeability, and chirality of the medium. In order to validate the definition of chiral nihility, we assume $\varepsilon=0, \mu=0$, and $\kappa \neq 0$ at certain frequency, which provides the constitutive relations as [4]

$$
\begin{aligned}
& \mathbf{D}=-j \kappa \sqrt{\epsilon_{0} \mu_{0}} \mathbf{H} \\
& \mathbf{B}=+j \kappa \sqrt{\epsilon_{0} \mu_{0}} \mathbf{E} .
\end{aligned}
$$

In chiral nihility mediums, there exist two (right- and left-) circularly polarized eigenwaves such that $\mathbf{E}=\mathbf{E}_{+}+\mathbf{E}_{-}$ and $\mathbf{H}_{+}= \pm\left(j / \eta_{1}\right)\left(\mathbf{E}_{ \pm}\right)$with wavenumbers as $k_{ \pm}= \pm \kappa k_{0}$ (a positive sign being the indication of right-circular and a negative sign for left-circular polarization). Also, $\eta_{1}$ is the medium impedance, given by $\lim _{\substack{\varepsilon \rightarrow 0 \\ \mu \rightarrow 0}}(\mu / \varepsilon)$. The two circularly polarized waves propagating through chiral nihility mediums contribute to backward waves. Apart from this, nihility mediums also exhibit the phenomenon of negative refraction/reflection.

Materials with such exotic features have drawn attention of many researchers as these offer potential applications in optics. Within the context, several alternate efforts have been made for the development of such materials $[5,6]$; references $[7,8]$ discuss the electromagnetic behavior of these.

Optical fibers embedded with conducting sheath helix structures at the core-clad interface allow altering the dispersion characteristics of these through properly adjusting the helix pitch angle [9-13]. Such helical structures have been in use in travelling wave tubes for the purpose of microwave amplification [14]. References [15, 16] report the dispersion behavior of helical clad elliptic fibers. It is noteworthy that elliptical waveguide has the distinct advantage that the transmission modes are more stable in these, and 
the attenuation modes are reduced as compared with its circular counterpart. In such guides, only hybrid modes exist to satisfy the boundary conditions [17].

The investigation of modified fiber structures with embedded conducting helix and nihility mediums would be interesting. As stated above, chiral nihility mediums have been greatly discussed in the literature [2]. As the nihility mediums have simultaneously vanishing values of permittivity and permeability, the motivation of the present paper comes from the idea of incorporating such medium into elliptical and cylindrical fiber geometries. It is well known that elliptical fibers have been of great use in signal detection in communication systems. However, in the present study, the emphasis has been given more on the dispersion properties that can be tailored through the incorporation of conducting sheath helical windings.

Iqbal et al. [18] reported before the dispersion features of helical clad fibers with chiral nihility mediums. The present communication remains a platform where we present a comparative study of fibers with elliptical and circular crosssections in which chiral nihility cylinders are encapsulated with infinitely extended dielectric clads in the transverse direction. We emphasize on the determination of effective refractive index (RI) as a function of normalized frequency for the fiber structures under consideration. The radial Mathieu function and its modified forms are incorporated for the determination of electric/magnetic fields in elliptical case [19], and the investigations are made corresponding to a few low-order hybrid modes under the situation of two extreme values of helix pitch angle.

\section{Formulation}

In the present analysis, we consider elliptical and circular fiber geometries with the loadings of conducting sheath helical windings with certain orientations at the core-clad interface. For instance, we consider the two extreme situations when the orientation becomes perpendicular and parallel to the optical axis. Furthermore, for both the fiber structures, the core section is composed of chiral nihility medium, and the clad region is linear, homogeneous, and isotropic dielectric medium. We now discuss the two fiber structures individually.

2.1. The Case of Elliptical Fiber. Figure 1(a) shows fiber structure of elliptical cross-section with the core of chiral nihility medium and dielectric clad. The structural geometry essentially approves the use of orthogonal elliptical coordinates $(\xi, \eta, z)$, as shown in Figure $1(\mathrm{~b})$. Here, $\xi$ represents contour surfaces of confocal elliptical cylinder and takes the value $0 \leq \xi \leq+\infty$, while $\eta$ represents confocal hyperbolic cylinders having range $0 \leq \xi \leq 2 \pi$. The core-clad boundary exists at constant confocal elliptic cylinder $\xi=\xi_{0}$. The distance between the two foci is $2 q=2 a e$, where $e$ is the eccentricity of elliptical cross-section, determined as $1 / \cosh \left(\xi_{0}\right)$, and $a$ is the semimajor axis of length $q \cosh \left(\xi_{0}\right)$ along the $x$-direction. Also, the semiminor axis along $y$-axis has the length $b=$ $q \sinh \left(\xi_{0}\right)$. Both the core and the clad regions are assumed to be nonmagnetic in nature; that is, $\mu_{1}=\mu_{2}=\mu_{0}$ (permeability of free-space).

Upon substituting the constitutive relations in (2a) and (2b) into Maxwell's equations, one obtains Helmholtz equations for longitudinal field components $E_{z}$ or $H_{z}$ in orthogonal elliptical coordinates as

$$
\left[\frac{\partial^{2}}{\partial \xi^{2}}+\frac{\partial^{2}}{\partial \eta^{2}}+2 p(\cosh 2 \xi-\cos 2 \eta)\right]\left[\begin{array}{c}
E_{z \pm} \\
H_{z \pm}
\end{array}\right]=0
$$

with

$$
\begin{gathered}
p=\frac{q^{2}\left(k_{ \pm}^{2}-\beta^{2}\right)}{4}, \\
k_{ \pm}= \pm \kappa k_{0}
\end{gathered}
$$

where $q=a e$ and $k_{0}$ is the free-space wave number. Ignoring the exponential term $\exp \{j(\omega t-\beta z)\}$, we can write the longitudinal and transverse field components [17] in the fiber core as

$$
\begin{gathered}
E_{z 1}=(A+B) S e_{m}\left(\xi, \gamma_{1}^{2}\right) \sin (m \eta) \\
H_{z 1}=\frac{j}{\eta_{1}}(A-B) S e_{m}\left(\xi, \gamma_{1}^{2}\right) \sin (m \eta) \\
E_{\xi 1}=(A+B)\left[-\frac{j \beta}{k_{r}^{2} q l} S e_{m}^{\prime}\left(\xi, \gamma_{1}^{2}\right) \sin (m \eta)\right. \\
\left.+\frac{\kappa k_{0} m}{k_{r}^{2} q l} S e_{m}\left(\xi, \gamma_{1}^{2}\right) \cos (m \eta)\right] \\
H_{\xi 1}=(A-B)\left[\frac{\beta}{k_{r}^{2} q l} S e_{m}^{\prime}\left(\xi, \gamma_{1}^{2}\right) \sin (m \eta)\right. \\
\left.+\frac{j \kappa k_{0} m}{\eta_{1} k_{r}^{2} q l} S e_{m}\left(\xi, \gamma_{1}^{2}\right) \cos (m \eta)\right] \\
+\frac{\kappa k_{0}}{k_{r}^{2} q l} S e_{m}^{\prime}\left(\xi, \gamma_{1}^{2}\right) \sin (m \eta) \\
E_{\eta 1}=(A+B) \\
\left.\quad-\frac{j \beta m}{k_{r}^{2} q l} S e_{m}\left(\xi, \gamma_{1}^{2}\right) \cos (m \eta)\right] \\
H_{\eta 1}=(A-B)\left[\frac{j \kappa k_{0}}{\eta_{1} k_{r}^{2} q l} S e_{m}^{\prime}\left(\xi, \gamma_{1}^{2}\right) \sin (m \eta)\right. \\
\left.+\left(\xi, \gamma_{1}^{2}\right) \cos (m \eta)\right]
\end{gathered}
$$

Similarly, the longitudinal and the transverse field components in the clad section will assume the forms as

$$
\begin{gathered}
E_{z 2}=(C+D) \operatorname{Gek}_{m}\left(\xi, \gamma_{2}^{2}\right) \sin (m \eta) \\
H_{z 2}=\frac{j}{\eta_{2}}(C-D) G e k_{m}\left(\xi, \gamma_{2}^{2}\right) \sin (m \eta)
\end{gathered}
$$




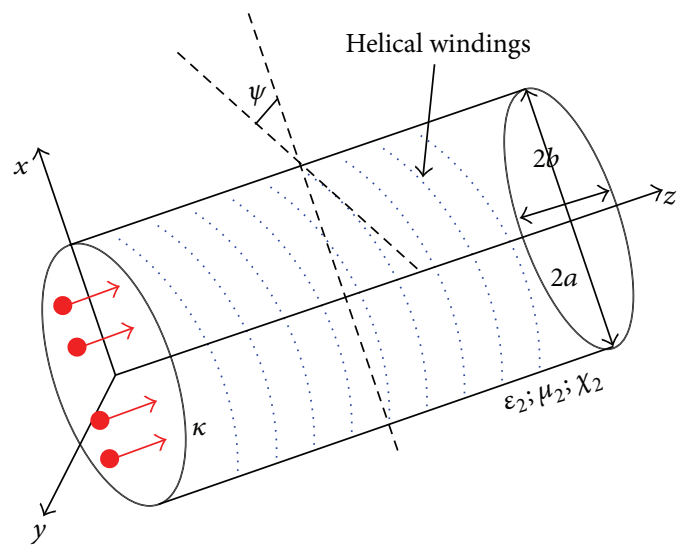

(a)

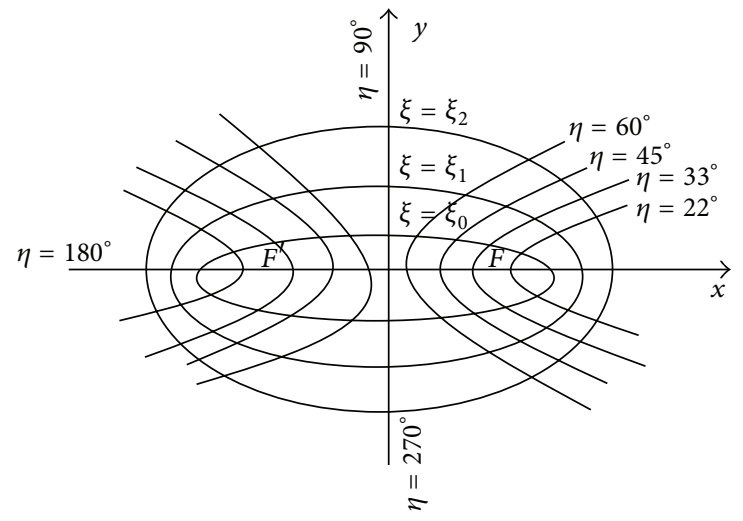

(b)

Figure 1: (a) Schematic of the conducting sheath helix embedded elliptical optical fiber with core-clad interface at $\xi=\xi_{0}$. (b) Orthogonal elliptic coordinate system with the semimajor and semiminor axes along the $x$-and $y$-directions, respectively.

$$
\begin{gathered}
E_{\xi 2}=\frac{1}{\tau^{2} q l}\left[-j \beta(C+D) G e k_{m}^{\prime}\left(\xi, \gamma_{2}^{2}\right) \sin (m \eta)\right. \\
\left.+\frac{m \mu_{0} \omega}{\eta_{2}}(C-D) G e k_{m}\left(\xi, \gamma_{2}^{2}\right) \cos (m \eta)\right] \\
H_{\xi 2}=\frac{1}{\tau^{2} q l}\left[j \omega \varepsilon_{2}(C+D) G e k_{m}\left(\xi, \gamma_{2}^{2}\right) \cos (m \eta)\right. \\
\left.+\frac{\beta}{\eta_{2}}(C-D) G e k_{m}^{\prime}\left(\xi, \gamma_{2}^{2}\right) \sin (m \eta)\right] \\
E_{\eta 2}=\frac{1}{\tau^{2} q l}\left[-j m \beta(C+D) G e k_{m}\left(\xi, \gamma_{2}^{2}\right) \cos (m \eta)\right. \\
\left.-\frac{\omega \mu_{0}}{\eta_{2}}(C-D) G e k_{m}^{\prime}\left(\xi, \gamma_{2}^{2}\right) \sin (m \eta)\right] \\
1 \\
H_{\eta 2}=-\frac{1}{\tau^{2} q l}\left[j \omega \varepsilon_{2}(C+D) G e k_{m}^{\prime}\left(\xi, \gamma_{2}^{2}\right) \sin (m \eta)\right. \\
\left.-\frac{m \beta}{\eta_{2}}(C-D) G e k_{m}\left(\xi, \gamma_{2}^{2}\right) \cos (m \eta)\right] .
\end{gathered}
$$

In (5a)-(5f) and (6a)-(6f), the subscripts 1 and 2 represent situations in the core and the clad sections, respectively. Also, $A, B, C$, and $D$ are unknown arbitrary constants that can be determined by using suitable boundary conditions, $m$ is the mode-designating parameter, and $\eta_{2}$ is the impedance of clad dielectric medium. Furthermore, $\operatorname{Se}_{m}\left(\xi, \gamma_{1}^{2}\right)$ is the radial Mathieu function and $\operatorname{Ge}_{m}\left(\xi, \gamma_{2}^{2}\right)$ is the modified radial Mathieu function along with the prime representing their derivatives with respect to the argument. The other used symbols have their meanings as follows:

$$
k_{r}=\sqrt{k_{ \pm}^{2}-\beta^{2}}=k_{0} \sqrt{( \pm \kappa)^{2}-\left(\frac{\beta}{k_{0}}\right)^{2}}
$$

$$
\begin{gathered}
\tau=k_{0} \sqrt{\left(\frac{\beta}{k_{0}}\right)^{2}-\left(n_{2}\right)^{2}} \\
\gamma_{1}=0.5 q \sqrt{\left(k_{ \pm}\right)^{2}-\beta^{2}}=0.5 q \sqrt{\left( \pm \kappa k_{0}\right)^{2}-\beta^{2}} \\
\gamma_{2}=0.5 q \sqrt{\beta^{2}-\left(k_{0} n_{2}\right)^{2}} \\
l=\sqrt{\cosh ^{2} \xi_{0}-\cos ^{2} \eta} .
\end{gathered}
$$

Here, the parameter $\beta$ represents propagation constant in the medium. Furthermore, it must be remembered that the core parameter $\gamma_{1}$ in (7c) above is related to the quantity $p$ in (4a) as $p=\gamma_{1}^{2}$. Now, we apply the boundary conditions at the corehelical clad interface present at the boundary $\xi=\xi_{0}$. These conditions require that tangential components of electric field are zero along the direction of conducting helix, while the tangential components of electric/magnetic fields are continuous in the direction perpendicular to conducting helix [9]. The implementation of boundary conditions provides a set of four equations, which would ultimately yield the values of the aforesaid unknown constants in terms of only one constant. The set of four equations gives the characteristic eigenvalue equation for the system from which the values of propagation constant $\beta$ can be determined:

$$
\begin{gathered}
\left(\frac{4}{l^{3} q^{3} \eta_{1} \eta_{2} \tau_{1}^{4} \tau_{2}^{2}}\right) \mu_{0} \omega \sin ^{2}(m \eta) G e k_{m}\left(\xi_{0}, \gamma_{2}^{2}\right) \operatorname{Gek}_{m}^{\prime}\left(\xi_{0}, \gamma_{2}^{2}\right) \\
\times\left[\kappa k_{0} \cos (\psi) \sin (m \eta) S e_{m}^{\prime}\left(\xi_{0}, \gamma_{1}^{2}\right)+S e_{m}\left(\xi_{0}, \gamma_{1}^{2}\right)\right. \\
\left.\times\left\{-j m \beta \cos (m \eta) \cos (\psi)+l q \tau_{1}^{2} \sin (m \eta) \sin (\psi)\right\}\right] \\
\times\left[\kappa k_{0} \cos (\psi) \sin (m \eta) S e_{m}^{\prime}\left(\xi_{0}, \gamma_{1}^{2}\right)-j S e_{m}\left(\xi_{0}, \gamma_{1}^{2}\right)\right. \\
\left.\times\left\{m \beta \cos (m \eta) \cos (\psi)+l q \tau_{1}^{2} \sin (m \eta) \sin (\psi)\right\}\right]=0 .
\end{gathered}
$$


Further, the boundary conditions yield the values of unknown constants $B, C$, and $D$ in (5a)-(5f) and (6a)-(6f) in terms of $A$ as

$$
\begin{gathered}
B=\left(-1+\frac{2 a_{7}\left(a_{5} a_{3}+a_{2} a_{6}\right)}{a_{7}\left(a_{5} a_{3}+a_{2} a_{6}\right)+a_{3} a_{4} a_{8}-a_{2} a_{4} a_{1}}\right) A \\
C=\left(\frac{2 a_{3} a_{4} a_{7}}{a_{7}\left(a_{5} a_{3}+a_{2} a_{6}\right)+a_{3} a_{4} a_{8}-a_{2} a_{4} a_{1}}\right) A \\
D=\left(\frac{2 a_{2} a_{4} a_{7}}{a_{7}\left(a_{5} a_{3}+a_{2} a_{6}\right)+a_{3} a_{4} a_{8}-a_{2} a_{4} a_{1}}\right) A .
\end{gathered}
$$

In (9), the used symbols have their meanings as follows:

$$
\begin{aligned}
& a_{1}=\frac{j}{\eta_{2}} G e k_{m}\left(\xi_{0}, \gamma_{2}^{2}\right) \sin (m \eta) \sin (\psi) \\
& +\frac{1}{\tau^{2} q l}\left\{j \omega \varepsilon_{2} G e k_{m}^{\prime}\left(\xi_{0}, \gamma_{2}^{2}\right) \sin (m \eta) \cos (\psi)\right. \\
& \left.+\frac{m \beta}{\eta_{2}} G e k_{m}\left(\xi_{0}, \gamma_{2}^{2}\right) \cos (m \eta) \cos (\psi)\right\} \\
& a_{2}=G e k_{m}\left(\xi_{0}, \gamma_{2}^{2}\right) \sin (m \eta) \sin (\psi) \\
& -\frac{1}{\tau^{2} q l}\left\{j m \beta G e k_{m}\left(\xi_{0}, \gamma_{2}^{2}\right) \cos (m \eta) \cos (\psi)\right. \\
& \left.+\frac{\omega \mu_{0}}{\eta_{2}} G e k_{m}^{\prime}\left(\xi_{0}, \gamma_{2}^{2}\right) \sin (m \eta) \cos (\psi)\right\} \\
& \left.-\frac{\omega \mu_{0}}{\eta_{2}} G e k_{m}^{\prime}\left(\xi_{0}, \gamma_{2}^{2}\right) \sin (m \eta) \cos (\psi)\right\} \\
& -\frac{1}{\tau^{2} q l}\left\{j m \beta G e k_{m}\left(\xi_{0}, \gamma_{2}^{2}\right) \cos (m \eta) \cos (\psi)\right. \\
& \left.\gamma_{2}^{2}\right) \sin (m \eta) \sin (\psi) \\
&
\end{aligned}
$$$$
a_{4}=S e_{m}\left(\xi_{0}, \gamma_{1}^{2}\right) \sin (m \eta) \cos (\psi)
$$$$
-\frac{1}{k_{r}^{2} q l}\left\{\kappa k_{0} S e_{m}^{\prime}\left(\xi_{0}, \gamma_{1}^{2}\right) \sin (m \eta) \sin (\psi)\right.
$$$$
\left.-j m \beta S e_{m}\left(\xi_{0}, \gamma_{2}^{2}\right) \cos (m \eta) \sin (\psi)\right\}
$$

$$
\begin{aligned}
a_{5}=\operatorname{Gek}_{m}\left(\xi_{0}, \gamma_{2}^{2}\right) \sin (m \eta) \cos (\psi) \\
+\frac{1}{\tau^{2} q l}\left\{j m \beta G e k_{m}\left(\xi_{0}, \gamma_{2}^{2}\right) \cos (m \eta) \sin (\psi)\right. \\
\left.+\frac{\omega \mu_{0}}{\eta_{2}} \operatorname{Gek}_{m}^{\prime}\left(\xi_{0}, \gamma_{2}^{2}\right) \sin (m \eta) \sin (\psi)\right\}
\end{aligned}
$$

$$
\begin{gathered}
a_{6}=\operatorname{Gek}_{m}\left(\xi_{0}, \gamma_{2}^{2}\right) \sin (m \eta) \cos (\psi) \\
+\frac{1}{\tau^{2} q l}\left\{j m \beta G e k_{m}\left(\xi_{0}, \gamma_{2}^{2}\right) \cos (m \eta) \sin (\psi)\right. \\
\left.-\frac{\omega \mu_{0}}{\eta_{2}} G e k_{m}^{\prime}\left(\xi_{0}, \gamma_{2}^{2}\right) \sin (m \eta) \sin (\psi)\right\} \\
a_{7}=\frac{j}{\eta_{1}}\left[S e_{m}\left(\xi_{0}, \gamma_{1}^{2}\right) \sin (m \eta) \sin (\psi)\right. \\
+\frac{1}{k_{r}^{2} q l}\left\{j \kappa k_{0} \operatorname{Se}_{m}^{\prime}\left(\xi_{0}, \gamma_{1}^{2}\right) \sin (m \eta) \cos (\psi)\right. \\
\left.\left.+m \beta S e_{m}\left(\xi_{0}, \gamma_{2}^{2}\right) \cos (m \eta) \cos (\psi)\right\}\right] \\
a_{8}=\frac{j}{\eta_{2}} G e k_{m}\left(\xi_{0}, \gamma_{2}^{2}\right) \sin (m \eta) \sin (\psi) \\
+\frac{1}{\tau^{2} q l}\left\{-j \omega \varepsilon_{2} G e k_{m}^{\prime}\left(\xi_{0}, \gamma_{2}^{2}\right) \sin (m \eta) \cos (\psi)\right. \\
\left.-\frac{m \beta}{\eta_{2}} G e k_{m}\left(\xi_{0}, \gamma_{2}^{2}\right) \cos (m \eta) \cos (\psi)\right\} .
\end{gathered}
$$

Equation (8) can be used to estimate the dispersion characteristics of elliptical core fiber structure with chiral nihility medium and embedded with conducting sheath helical windings at the core-clad interface.

2.2. The Case of Circular Fiber. Following the similar procedure as adopted for the case of elliptical fiber, the expressions for transverse and longitudinal components of electric/magnetic fields may be obtained from [20]. However, the values of unknown constants can be determined by using suitable field continuity conditions at the helical interface present at the core/clad boundary [9]. This would provide a set of four simultaneous linear equations for the unknown coefficients, the nontrivial solution to which would be obtained by setting the determinant formed by the coefficients (of the unknown constants) to zero. This would ultimately provide the following characteristic eigenvalue equation:

$$
\begin{gathered}
\frac{1}{a^{2} k_{r}^{4} \eta_{1} \tau}\left[\left\{4 j k_{2} K_{m}(a \tau) K_{m}^{\prime}(a \tau)\left(\cos ^{2} \psi+\sin ^{2} \psi\right)\right\}\right. \\
\times\left\{m \beta J_{m}\left(a k_{r}\right) \cos \psi+a k_{r}^{2} J_{m}\left(a k_{r}\right) \sin \psi\right. \\
\left.\left.-a \kappa k_{0} k_{r} J_{m}^{\prime}\left(a k_{r}\right) \cos \psi\right\}^{2}\right]=0 .
\end{gathered}
$$

The above equation is representing dispersion relation of system under consideration where $J_{m}(\bullet)$ and $K_{m}(\bullet)$ are, respectively, Bessel and the modified Bessel functions; prime being the representation of their derivatives with respect to the argument. Also, $k_{2}=\omega \sqrt{\varepsilon_{2} \mu_{0}}$, and $\kappa$ is the chirality parameter; the other symbols being defined similarly as stated above. Equation (11) can be used to obtain the propagation constants of sustained modes. 
In this case too, the values of (four) unknown constants $A_{c}, B_{c}, C_{c}$, and $D_{c}$ (say) can be determined by following the procedure as discussed above for the case of elliptical fiber. Finally, it can be shown that the values of $B_{c}, C_{c}$, and $D_{c}$ can be written in terms of $A_{c}$ as follows:

$$
\begin{gathered}
B_{c}=\left[-1+\frac{2 b_{7}\left(b_{5} b_{3}+b_{2} b_{6}\right)}{b_{7}\left(b_{5} b_{3}+b_{2} b_{6}\right)+b_{3} b_{4} b_{8}-b_{2} b_{4} b_{1}}\right] A_{c} \\
C_{c}=\frac{2 b_{3} b_{4} b_{7}}{b_{7}\left(b_{5} b_{3}+b_{2} b_{6}\right)+b_{3} b_{4} b_{8}-b_{2} b_{4} b_{1}} A_{c} \\
D_{c}=\frac{2 b_{2} b_{4} b_{7}}{b_{7}\left(b_{5} b_{3}+b_{2} b_{6}\right)+b_{3} b_{4} b_{8}-b_{2} b_{4} b_{1}} A_{c} .
\end{gathered}
$$

In these equations, the used symbols have their meanings as follows:

$$
\begin{aligned}
& b_{1} \\
& =\frac{j}{\eta_{2}}\left[K_{m}(\tau a) \sin \psi-\left\{\frac{\beta m}{\tau^{2} a} K_{m}(\tau a)+\frac{k_{2}}{\tau} K_{m}^{\prime}(\tau a)\right\} \cos \psi\right] \\
& b_{2}=K_{m}(\tau a) \sin \psi+\left\{-\frac{\beta m}{\tau^{2} a} K_{m}(\tau a)+\frac{k_{2}}{\tau} K_{m}^{\prime}(\tau a)\right\} \cos \psi \\
& b_{3}=-K_{m}(\tau a) \sin \psi+\left\{\frac{\beta m}{\tau^{2} a} K_{m}(\tau a)+\frac{k_{2}}{\tau} K_{m}^{\prime}(\tau a)\right\} \cos \psi \\
& b_{4}=J_{m}\left(k_{r} a\right) \cos \psi-\left\{\frac{\beta m}{a k_{r}^{2}} J_{m}\left(k_{r} a\right)-\frac{\kappa k_{0}}{k_{r}} J_{m}^{\prime}\left(k_{r} a\right)\right\} \cos \psi \\
& b_{5}=K_{m}(\tau a) \cos \psi+\left\{\frac{\beta m}{\tau^{2} a} K_{m}(\tau a)-\frac{k_{2}}{\tau} K_{m}^{\prime}(\tau a)\right\} \sin \psi \\
& b_{6}=K_{m}(\tau a) \cos \psi+\left\{\frac{\beta m}{\tau^{2} a} K_{m}(\tau a)+\frac{k_{2}}{\tau} K_{m}^{\prime}(\tau a)\right\} \sin \psi \\
& b_{8}=\frac{j}{\eta_{2}}\left[K_{m}(\tau a) \sin \psi\right. \\
& \eta_{1} \\
& b_{1}
\end{aligned}
$$

\section{Results and Discussion}

We now focus on the dispersion characteristics of fiber structures, which are greatly governed by the pitch angle of helical windings. For computations, we choose two extreme values of pitch angle as $0^{\circ}$ and $90^{\circ}$. The other fiber parameters are the core chirality parameter $\kappa$ as 1.55 and 1.46 , the clad RI $n_{2}$ as 1.49 (acrylic glass) and 1.444 (silica), the operating wavelength $\lambda$ as $1.55 \mu \mathrm{m}$, semimajor (a) and semiminor (b) axes lengths as $10 \mu \mathrm{m}$ and $7.6 \mu \mathrm{m}$ (in the elliptical case), respectively (for circular geometry the parameter $a$ is assumed to be the radius of circular cross-section), and the impedance of chiral nihility medium (in the core section) as equal to that of free-space. Under the scenario of hybrid $\mathrm{EH}$ mode excitation, computations are made for the values of mode index $m$ as 0,1 , and 2 .

Figures 2 and 3 and Figures 4 and 5, respectively, correspond to the situations of elliptical and circular core fibers, wherein the effective RI $\left(n_{\text {eff }}\right)$ is plotted against the normalized frequency parameter $V$. Considering the three low-order hybrid modes $E H_{01}, E H_{11}$, and $E H_{21}$, plots are made for $0^{\circ}$ and $90^{\circ}$ helix pitch angles along with the consideration of the core chirality parameter $\kappa$ as 1.55 and 1.46, and the clad RI $n_{2}$ as 1.49 and 1.444. Within the context, it remains noteworthy that the behavior of chiral nihility mediums is frequency dependent.

Figures 2(a) and 2(b), respectively, illustrate the dispersion characteristics of elliptical fiber having the characteristic helix pitch angles as $0^{\circ}$ and $90^{\circ}$ under the consideration of the three low-order hybrid modes $E H_{01}, E H_{11}$, and $E H_{21}$. We find that, in both the situations, the values of $n_{\text {eff }}$ generally decrease with the increase in mode order. We observe from Figure 2(a) that, under the situation when the conducting helical windings are perpendicular to the direction of wave propagation, the $E H_{01}, E H_{11}$, and $E H_{21}$ modes exhibit approximate cutoff values at $V \leq 3.726, V \leq 4.052$, and $V \leq$ 4.99 , respectively. As such, the lowest $E H_{01}$ mode shows the least cutoff. After the cutoff $V$-values, the $n_{\text {eff }}$ for all the modes gradually increase with the increase in $V$. Furthermore, the peaks and dips observed in the $n_{\text {eff }}-V$ plots corresponding to every mode demonstrate, respectively, the presence of slowand fast-waves in the fiber structure. In this respect, the $E H_{01}$ mode contributes more strongly to the slow- and fast-waves in the vicinity of $V \cong 3.8$ and $V \cong 4.4$.

The case of parallel orientation (i.e., $\psi=90^{\circ}$ ) of conducting helix is shown in Figure 2(b), wherein we observe that the $E H_{01}$ mode does not exhibit any cutoff within the specified frequency range $(V=1.547$, as obtained by the use of [17]). For the next $E H_{11}$ and $E H_{21}$ modes, the cutoff $V$ values exist at $V=4.004$ and $V=6.451$, respectively. As such, we find that a change in conducting helix orientation causes altering the dispersion behavior, and particularly, the longitudinal orientation of helix structure has the tendency to reduce the modal cutoffs, except for the mode $E H_{21}$ for which the cutoff is increased. We also notice that the $E H_{01}$ mode contributes more strongly to the slow- and fast-waves near $V \cong 3.2$ and $V \cong 4.9$ than the other two higher modes.

Considering $\kappa=1.46$ and $n_{2}=1.444$, and keeping the other operating conditions as the same, the results are illustrated in Figures 3(a) and 3(b). We observe that, for a $0^{\circ}$ helix pitch, the cutoff frequency increases for the $E H_{01}$ mode $(V=2.599)$, and it is decreased corresponding to the other higher order modes under consideration. It can be noticed that the cutoff frequencies occur at $V=2.665$ and $V=2.819$ corresponding to the $E H_{11}$ and the $E H_{21}$ modes, respectively. Furthermore, the surface modes occur at the normalized frequencies $V>4.5, V>4.831$, and $V>$ 4.925 corresponding to the $E H_{01}, E H_{11}$, and $E H_{21}$ modes, respectively. On the other hand, for a $90^{\circ}$ helix pitch angle, the cutoff frequency increases corresponding to the $E H_{21}$ mode only, and it gets decreased for the other modes at this value of pitch. Apart from these, the results demonstrate that 


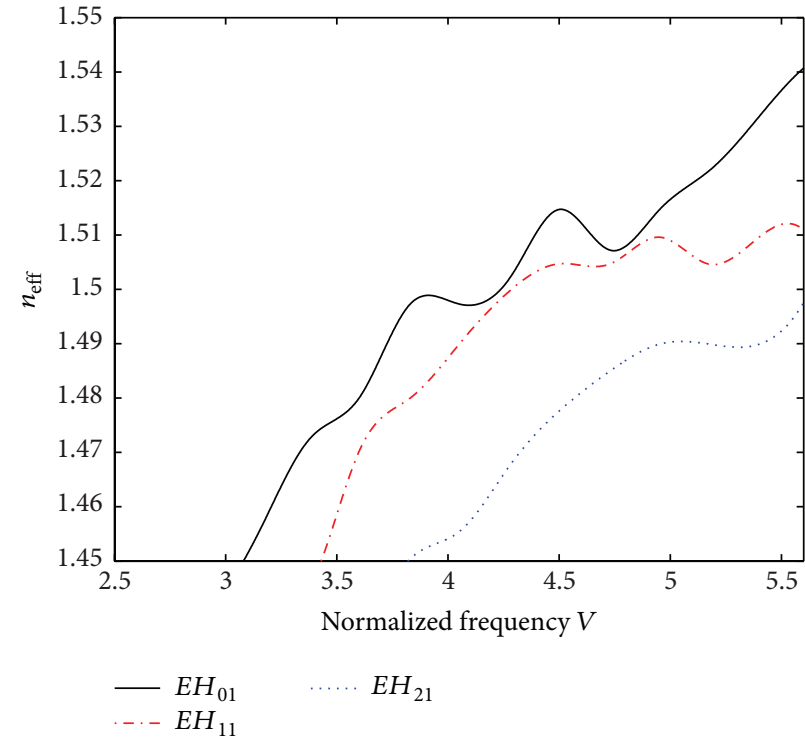

(a)

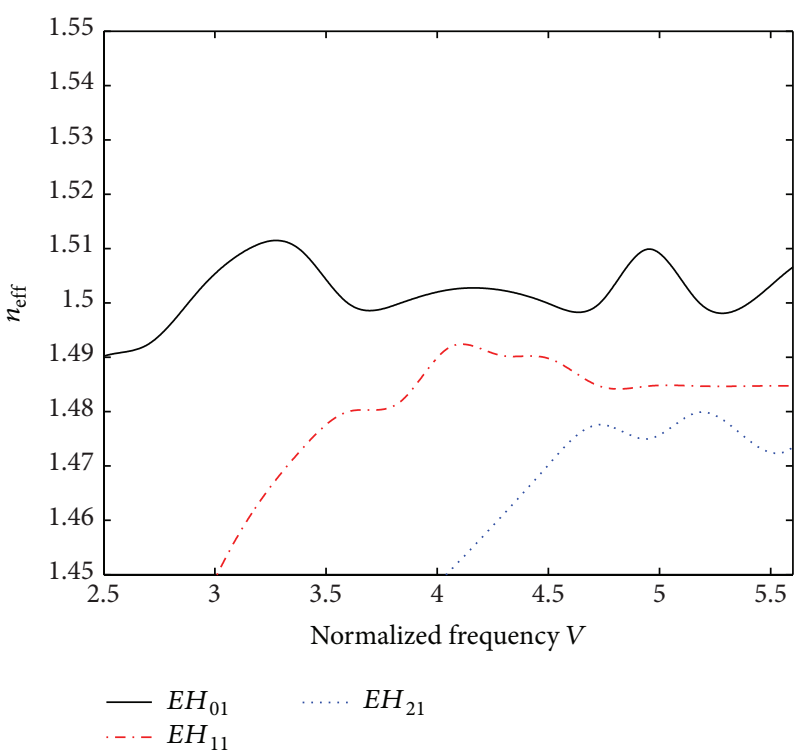

(b)

FIgURE 2: (a) Plot of $n_{\text {eff }}$ versus $V$ for elliptical fiber with pitch angle $0^{\circ}$ corresponding to $\kappa=1.55$ and $n_{2}=1.49$. (b) Plot of $n_{\text {eff }}$ versus $V$ for elliptical fiber with pitch angle $90^{\circ}$ corresponding to $\kappa=1.55$ and $n_{2}=1.49$.

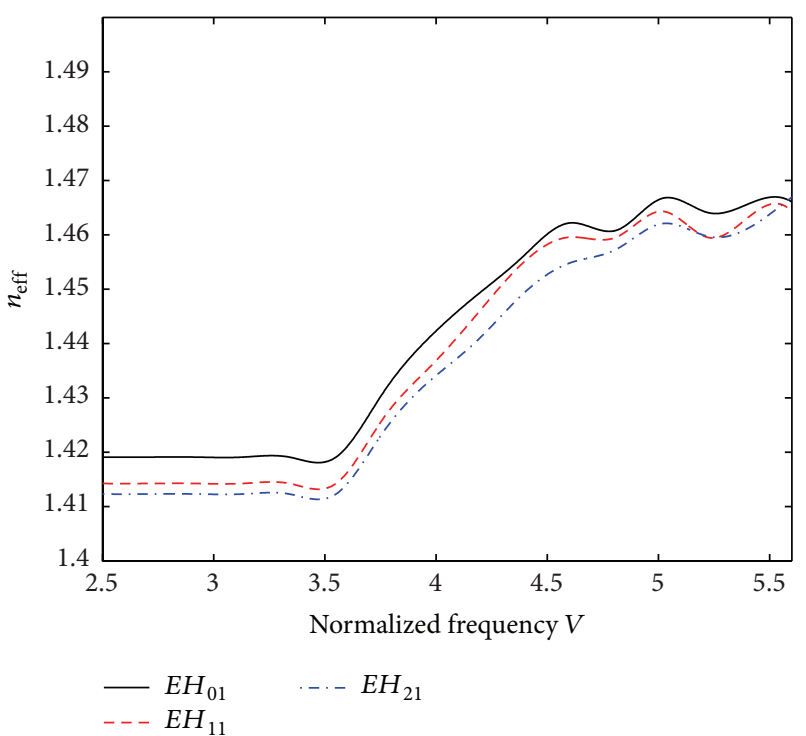

(a)

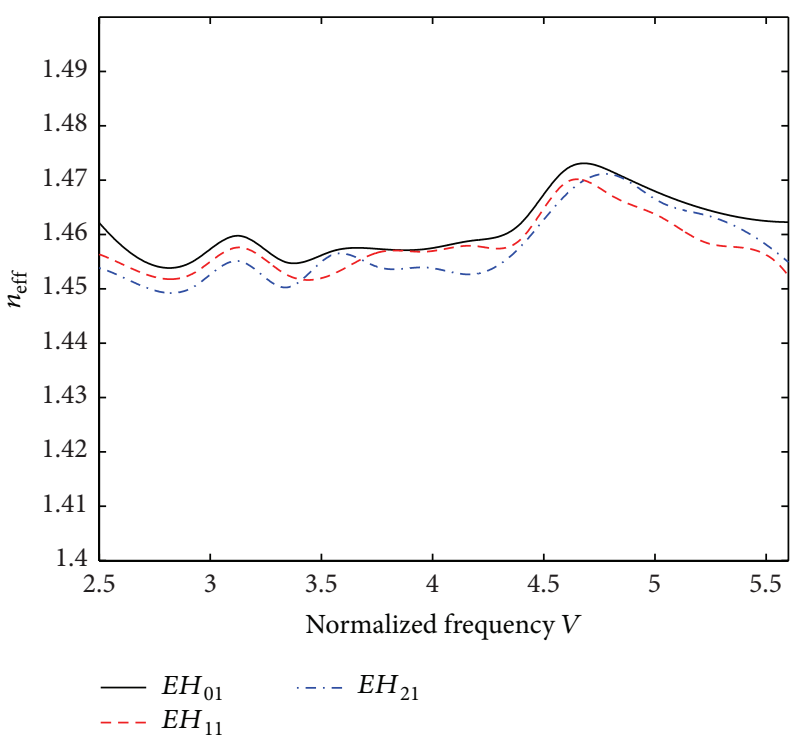

(b)

Figure 3: (a) Plot of $n_{\text {eff }}$ versus $V$ for elliptical fiber with pitch angle $0^{\circ}$ corresponding to $\kappa=1.46$ and $n_{2}=1.444$. (b) Plot of $n_{\text {eff }}$ versus $V$ for elliptical fiber with pitch angle $90^{\circ}$ corresponding to $\kappa=1.46$ and $n_{2}=1.444$.

a $90^{\circ}$ helix pitch makes the surface modes to occur at lower frequencies than what have been observed corresponding to a $0^{\circ}$ pitch.

Figures 4(a) and 4(b) correspond to the dispersion situations of fiber with circular cross-section when the conducting helix pitch angles assume the values $0^{\circ}$ and $90^{\circ}$, respectively. Similarly to the case of elliptical fiber (as discussed above), we consider the three low-order hybrid modes $E H_{01}, E H_{11}$, and $E H_{21}$, and we find that, in this case as well, the values of $n_{\text {eff }}$ generally increase with the decrease in mode order. We observe from Figure 4(a) that, when the helical windings are oriented in the direction perpendicular to the direction of propagation, all the stated modes exhibit cutoffs at $V \leq 1.956$, $V \leq 2.672$, and $V \leq 3.153$. Furthermore, corresponding to the $E H_{01}$ mode, a strong dip in the $n_{\text {eff }}-V$ plot near $V \cong 2.75$ exhibits the presence of slow- and fast-waves in the fiber. The other higher modes also contribute to such situations, but those are not as strong as that reflected by the $\mathrm{EH}_{01}$ mode. 


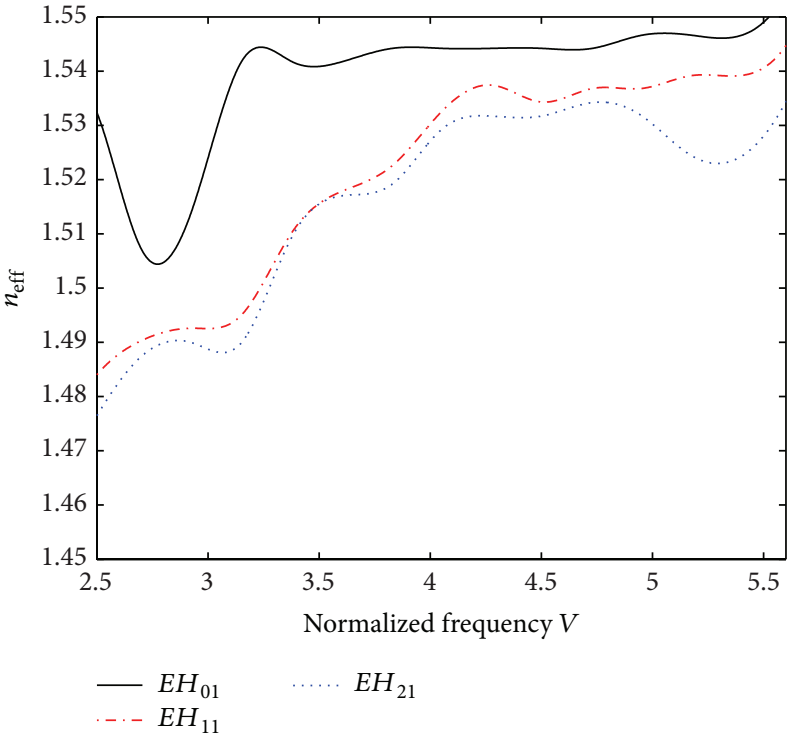

(a)

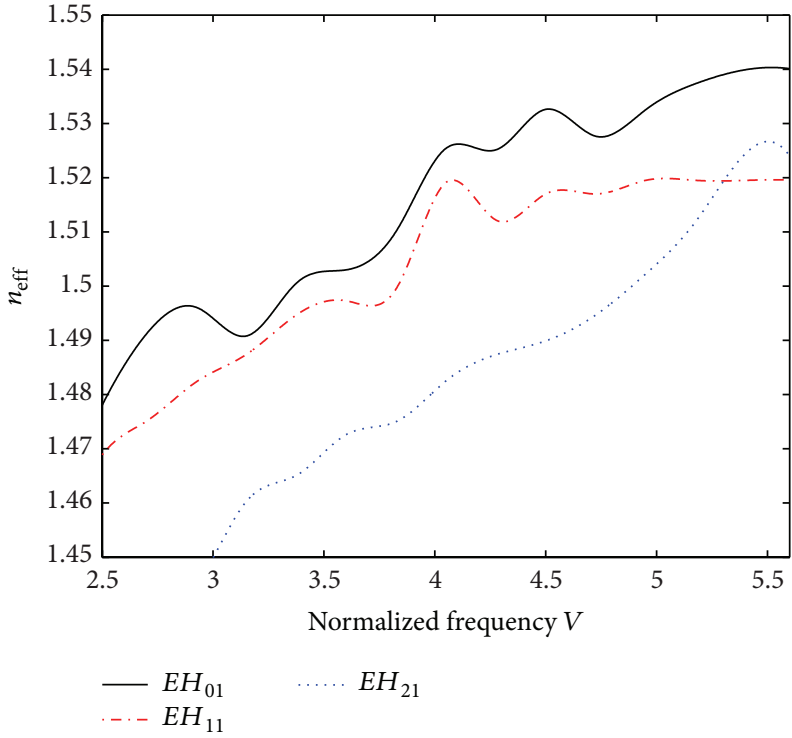

(b)

Figure 4: (a) Plot of $n_{\text {eff }}$ versus $V$ for circular fiber with pitch angle $0^{\circ}$ corresponding to $\kappa=1.55$ and $n_{2}=1.49$. (b) Plot of $n_{\text {eff }}$ versus $V$ for circular fiber with pitch angle $90^{\circ}$ corresponding to $\kappa=1.55$ and $n_{2}=1.49$.

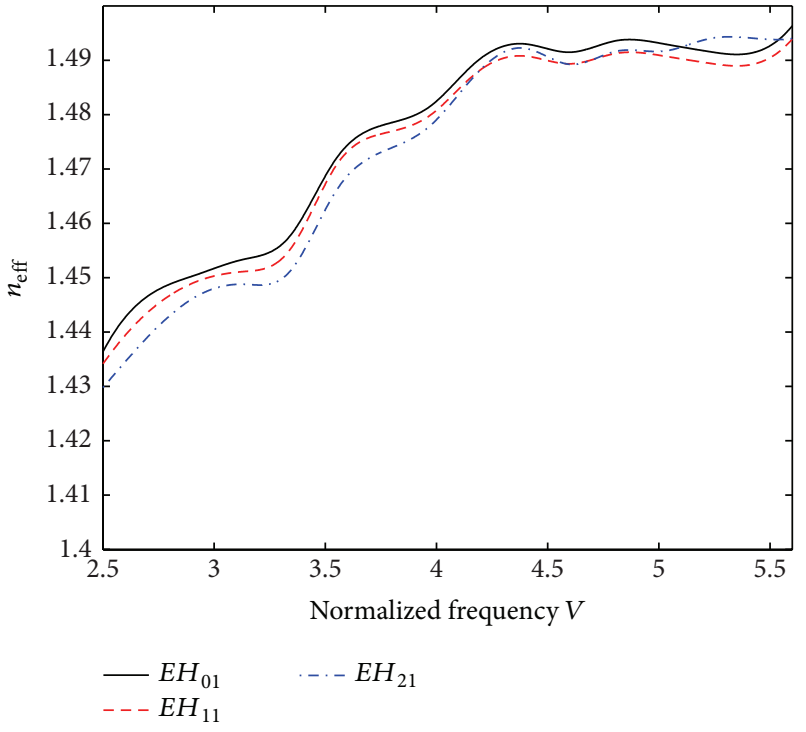

(a)



(b)

Figure 5: (a) Plot of $n_{\text {eff }}$ versus $V$ for circular fiber with pitch angle $0^{\circ}$ corresponding to $\kappa=1.46$ and $n_{2}=1.444$. (b) Plot of $n_{\text {eff }}$ versus $V$ for circular fiber with pitch angle $90^{\circ}$ corresponding to $\kappa=1.46$ and $n_{2}=1.444$.

Increasing the helix pitch angle to $90^{\circ}$, dispersion plots corresponding to all the low-order hybrid modes are shown in Figure 4(b). We observe from this figure that cutoff frequencies of $E H_{01}, E H_{11}$, and $E H_{21}$ modes occur at $V \leq 2.677$, $V \leq 3.226$, and $V \leq 4.512$, respectively. Furthermore, the conditions of slow- and fast-waves are better observed for the $E H_{01}$ and $E H_{11}$ modes for certain values of $V$. Comparing the dispersion behavior of circular fiber with that of the elliptical fiber, we notice that the average value of $n_{\text {eff }}$ remains a little larger in the case of the former one, and the highest value of $n_{\text {eff }}$ is attained when the helical windings are oriented perpendicular to the direction of wave propagation. Apart from this, the condition very close to mode degeneracy is observed for such helical orientation in fiber with circular cross-section, which is not found in the case of elliptical fiber.

Taking into account $\kappa=1.46$ and $n_{2}=1.444$, the obtained results are shown in Figures 5(a) and 5(b). We notice that, corresponding to a $0^{\circ}$ angle of helix pitch, the cutoff frequency is increased for the $E H_{01}$ and the $E H_{11}$ modes, and it is decreased for the $E H_{21}$ mode. Increasing the pitch to $90^{\circ}$, the cutoff frequency is decreased for all the stated modes. Furthermore, the effect due to the alteration of pitch angle on 
the surface modes can also be observed from Figures 5(a) and 5(b) (i.e., corresponding to $0^{\circ}$ pitch angle, $V>3.389\left(E H_{01}\right)$, $V>3.417\left(E H_{11}\right)$, and $V>3.471\left(E H_{21}\right)$, and corresponding to $90^{\circ}$ pitch, $V>4.817\left(E H_{01}\right), V>4.874\left(E H_{11}\right)$, and $\left.V>4.875\left(E H_{21}\right)\right)$.

In the foregoing investigation, results are obtained for different values of chirality parameter in the fiber core and the RI values of the fiber clad taking into account the cases of circular and elliptical fibers. From the respective plots, we can analyze the cutoffs for the guided and surface modes. In [20], cutoff and surface modes for the $E H_{01}$ mode occur at the normalized frequencies $V=2.405$ and $V=$ 1.332 , respectively. In the present investigation, however, corresponding to the chirality parameter in core $\kappa=1.55$ and the RI value of clad $n_{2}=1.49$, cutoffs occur at $V=1.956$ (for the pitch angle $0^{\circ}$ ) and $V=2.677$ (for the pitch angle $90^{\circ}$ ), and these cutoff frequencies show increase to $V=2.599$ (for the pitch angle $0^{\circ}$ ) and $V=3.841$ (for the pitch angle $90^{\circ}$ ) with the variation in system parameters (i.e., the core chirality $\kappa=1.46$ and the clad RI of clad $\left.n_{2}=1.444\right)$. No surface mode has been found to exist in the case of parametric values $\kappa=1.55$ and $n_{2}=1.49$; these occur corresponding to the situation of choosing $\kappa=1.46, n_{2}=$ 1.444 instead. For the other two modes $\left(E H_{11}\right.$ and $\left.E H_{-11}\right)$ in [20], cutoff frequencies are reported to occur at $V=0$ (for the $E H_{11}$ mode) and $V=3.832$ (for the $E H_{-11}$ mode), whereas no surface modes exist. However, in present analysis, cutoff frequencies (corresponding to the cases of $\kappa=1.55,1.46$, $\left.n_{2}=1.49,1.444\right)$ and surface modes (corresponding to $\kappa=$ $\left.1.46, n_{2}=1.444\right)$ are investigated for these modes $\left(E H_{11}\right.$ and $\left.E H_{21}\right)$, as described earlier.

\section{Conclusion}

The aforesaid discussion is pivoted to the role of conducting sheath helix embedded in chiral nihility fibers with circular and elliptical cross-sections. Considering the three low-order hybrid modes, analyses are made of the dispersion characteristics under the circumstances when the helical windings assume orientations perpendicular as well as parallel to the direction of wave propagation. It has been found that the helix orientations greatly affect the cutoff characteristics of modes. In certain situations, modes in circular fiber do not exhibit any cutoff, and also, the perpendicular orientation of conducting sheath helix in these brings in the condition close to mode degeneracy - the situation not observed for elliptical fibers. It is also found that the modal cutoff values for elliptical fiber are generally higher than those observed for circular fiber. Dispersion characteristics clearly indicate the existence of slow- and fast-waves in the fiber structure depending on the structural geometry as well as the helix orientations.

\section{Conflict of Interests}

The authors declare that there is no conflict of interests regarding the publication of this paper.

\section{Acknowledgments}

The authors are thankful to the two anonymous reviewers for making constructive criticisms on the paper. Also, they gratefully acknowledge the Ministry of Higher Education (Malaysia) for providing financial support to the work.

\section{References}

[1] S. Bassiri, C. H. Papas, and N. Engheta, "Electromagnetic wave propagation through a dielectric-chiral interface and through a chiral slab," Journal of the Optical Society of America A, vol. 5, no. 9, pp. 1450-1459, 1988.

[2] S. Tretyakov, A. Sihvola, and L. Jylhä, "Backward-wave regime and negative refraction in chiral composites," Photonics and Nanostructures-Fundamentals and Applications, vol. 3, no. 23, pp. 107-115, 2005.

[3] P. K. Choudhury and T. Yoshino, "Dependence of optical power confinement on core/cladding chiralities in chirofibers," Microwave and Optical Technology Letters, vol. 32, no. 5, pp. 359-364, 2002.

[4] S. Tretyakov, I. Nefedov, A. Sihvola, S. Maslovski, and C. Simovski, "Waves and energy in chiral nihility", Journal of Electromagnetic Waves and Applications, vol. 17, no. 5, pp. 695706, 2003.

[5] D. R. Smith and N. Kroll, "Negative refractive index in lefthanded materials," Physical Review Letters, vol. 85, no. 14, pp. 2933-2936, 2000.

[6] A. Lakhtakia, "On perfect lenses and nihility," International Journal of Infrared and Millimeter Waves, vol. 23, no. 3, pp. 339343, 2002.

[7] C.-W. Qiu, H.-Y. Yao, L.-W. Li, S. Zouhdi, and T.-S. Yeo, "Backward waves in magnetoelectrically chiral media: propagation, impedance, and negative refraction," Physical Review B: Condensed Matter and Materials Physics, vol. 75, no. 15, Article ID 155120, 2007.

[8] Q. Cheng, T. J. Cui, and C. Zhang, "Waves in planar waveguide containing chiral nihility metamaterial," Optics Communications, vol. 276, no. 2, pp. 317-321, 2007.

[9] K. Y. Lim, P. K. Choudhury, and Z. Yusoff, "Chirofibers with helical windings - an analytical investigation," Optik, vol. 121, no. 11, pp. 980-987, 2010.

[10] M. Ghasemi and P. K. Choudhury, "On the sustainment of optical power in twisted clad dielectric cylindrical fibers," Journal of Electromagnetic Waves and Applications, vol. 27, no. 11, pp. 1382-1391, 2013.

[11] M. Ghasemi and P. K. Choudhury, "Propagation through complex structured liquid crystal optical fibers," Journal of Nanophotonics, vol. 8, no. 1, Article ID 083997, 2014.

[12] M. Ghasemi and P. K. Choudhury, "Waves in tape helix loaded liquid crystal optical fiber," in Nanostructured Thin Films VII, vol. 9172 of Proceedings of SPIE, pp. 1-8, San Diego, Calif, USA, 2014.

[13] M. Ghasemi and P. K. Choudhury, "A revisit to the propagation through conducting helix loaded dielectric elliptic optical waveguides," International Journal of Microwave and Optical Technology, vol. 9, no. 1, pp. 119-123, 2014.

[14] J. R. Pierce, Travelling Wave Tubes, D. Van Nostrand, Princeton, NJ, USA, 1950.

[15] D. Kumar and O. N. Singh II, "Modal characteristic equation and dispersion curves for an elliptical step-index fiber with a 
conducting helical winding on the core-cladding boundary-an analytical study," Journal of Lightwave Technology, vol. 20, no. 8, pp. 1416-1424, 2002.

[16] D. Kumar, P. K. Choudhury, and F. A. Rahman, "Low eccentricity elliptical fibers with helical windings under slow-wave consideration-some special cases," Optik, vol. 121, no. 10, pp. 926-933, 2010.

[17] C. Yeh and F. Shimabukuro, The Essence of Dielectric Waveguides, chapter 6, Springer, New York, NY, USA, 2008.

[18] N. Iqbal, M. A. Baqir, and P. K. Choudhury, "Waves in microstructured conducting sheath helix embedded optical guides with chiral nihility and chiral materials," Journal of Nanomaterials, vol. 2014, Article ID 362739, 7 pages, 2014.

[19] M. J. Adams, An Introduction to Optical Waveguides, John Wiley \& Sons, London, UK, 1981.

[20] J. Dong, "Exotic characteristics of power propagation in the chiral nihility fiber," Progress in Electromagnetics Research, vol. 99, pp. 163-178, 2009. 

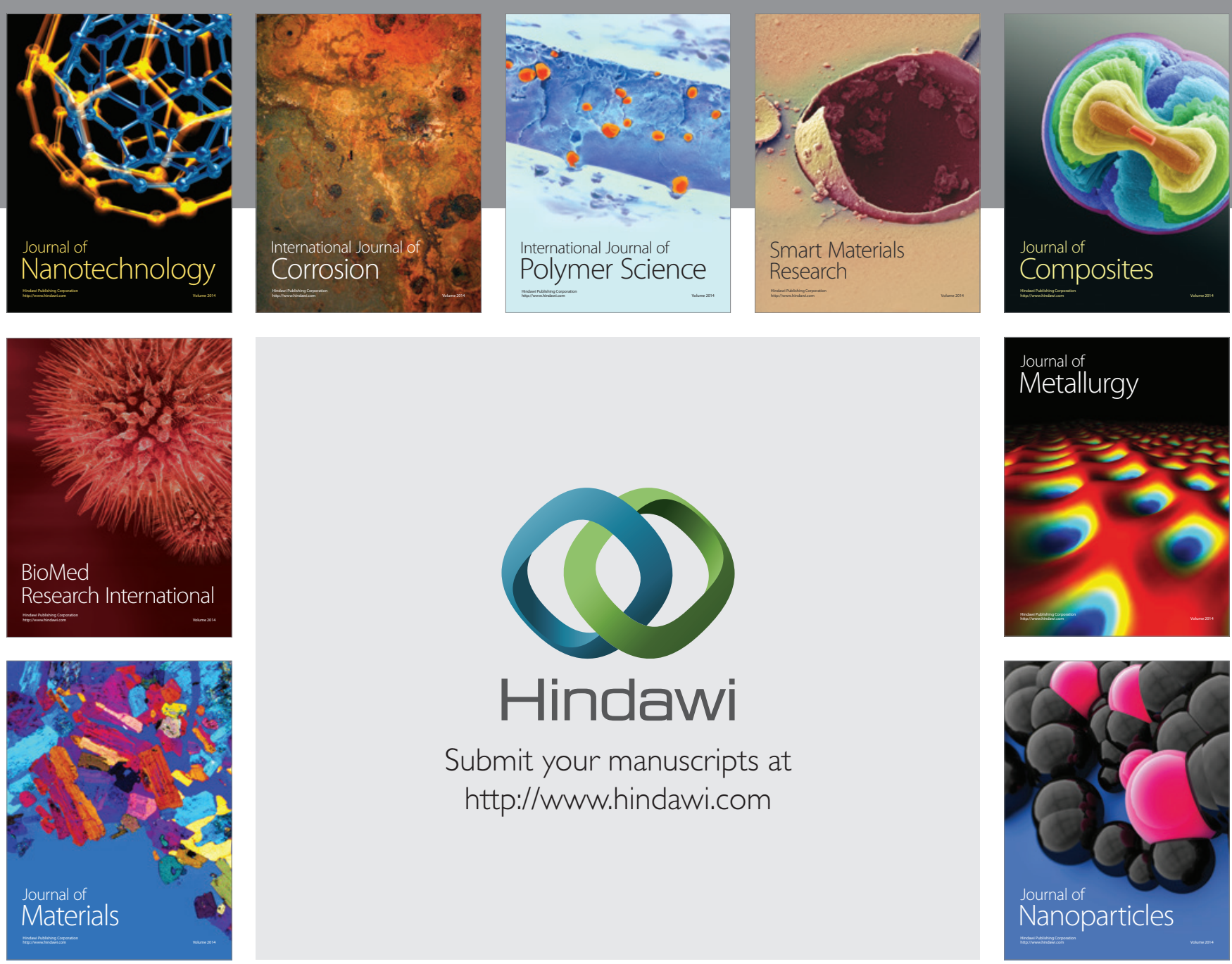

Submit your manuscripts at http://www.hindawi.com
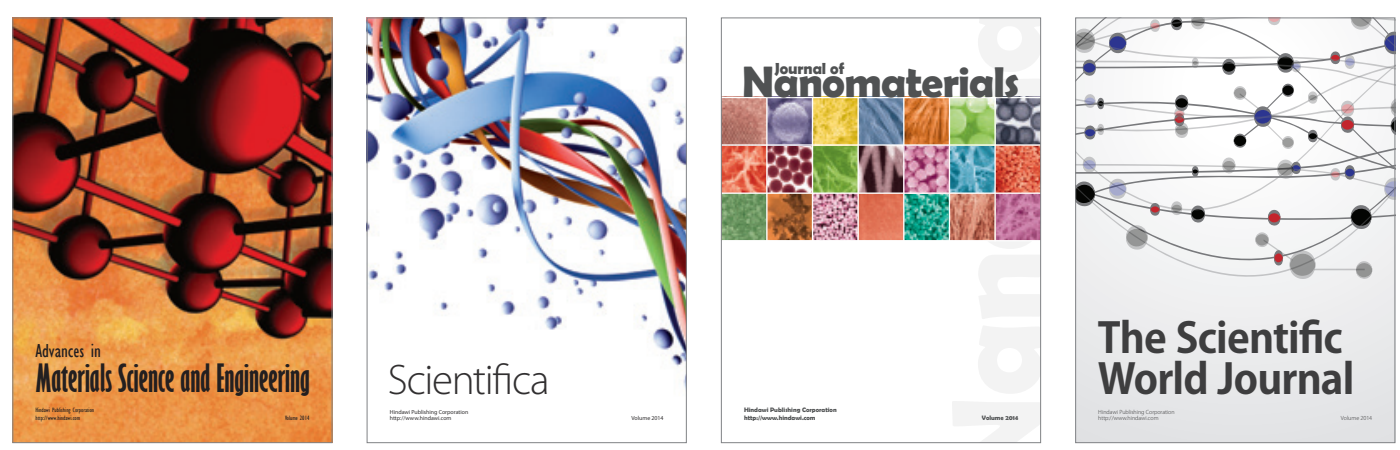

\section{The Scientific World Journal}
